\title{
ReVIEWS ON Physically BASED CONTROLLABLE FLUID ANIMATION
}

\section{Saithip Limtrakul ${ }^{1 *}$, Wisut Hantanong ${ }^{1}$ Pizzanu Kanongchaiyos ${ }^{1}$ and Tomoyuki Nishita ${ }^{2}$}

\author{
1 Department of Computer Engineering, Faculty of Engineering, \\ Chulalongkorn University, Thailand 10330 \\ ${ }^{2}$ Department of Complexity Science and Engineering, \\ Graduate School of Frontier Sciences, The University of Tokyo, Japan \\ E-mail: saithip_I@hotmail.com*,wizzup@wizzup.com¹, \\ pizzanu@cp.eng.chula.ac.th ${ }^{1}$ and nis@is.s.u-tokyo.ac.jp ${ }^{2}$
}

\section{ABSTRACT}

In computer graphics animation, animation tools are required for fluid-like motions which are controllable by users or animator, since applying the techniques to commercial animations such as advertisement and film. Many developments have been proposed to model controllable fluid simulation with the need in realistic motion, robustness, adaptation, and support more required control model. Physically based models for different states of substances have been applied in general in order to permit animators to almost effortlessly create interesting, realistic, and sensible animation of natural phenomena such as water flow, smoke spread, etc. In this paper, we introduce the methods for simulation based on physical model and the techniques for control the flow of fluid. We then discuss the existing control methods within three performances; control ability, realism, and computation time. Finally, we give a brief of the current and trend of the research areas.

\section{KEYWORDS}

controllable fluid animation, Naveir-Stokes' equations, smoothed particle hydrodynamics, fluid simulation 


\section{Introduction}

In our everyday lives, fluids can be found everywhere. Its existence is directly related to our routines; for example, the lukewarm vapor of hot coffee, the ripples of river's surface, and the disastrous smoke of a fire. The characteristics of fluids are, in a sense, useful in both education and entertainments fields. We require tools or simulators for simulating the fluid phenomena in order to estimate or predict the results from its movement. In computer graphics animation, a realistic simulation of natural phenomena has been developed continuously for several decades. Formerly; in computer animation, the animators simulate the fluid movement by using non-physically based method; do not solving the governing equations of fluid dynamics. Nevertheless, people have higher requirements on the visual effect of animation. The artists or animators have to spend much time on the complex scene. Various varying movements of fluid are difficult for the animators to simulate frame by frame. For this reason, physically based models for different states of fluid; which are describes by Navier-Stokes' equations, have been applied in general to solve the problems. Unlike key frame or procedural based techniques, physical model allows animators to effortlessly create fluid phenomena due to its governing equation which describes the fluid behaviors. Hence, this method became the alternative approach for creating realistic fluid animations.

In the field of computer graphics, fluid simulations require far less precision than those used in physics field. Animators require the systems or tools that can be easily implemented, less computation, and give them believable result or look fairly good. So, we assume incompressible fluid; the volume of fluid in the system is constant throughout the time, and homogeneous fluid which its density is constant, for the simulation. The combination of incompressibility and homogeneity leads the fluid whose density and temperature are nearly constant. The assumptions are common in fluid dynamics because fluids regularly move at low speeds, whereas high speed moving phenomena are governed by other equations.

Furthermore, animators not only need realistic animation, but also need a desirable fluids dynamics. They would like to control fluid behaviors as their imagination, since applying the desired fluid movement to commercial animations such as in the movie and advertisements. For decades, many developments have been proposed to model controllable fluid simulation with the need in realistic motion, robustness, adaptation, and support more required control model. With both contributions of physically based model and control method, imaginary complex movement of fluid is ready for simulation.

In this paper, so we take a survey on controllable fluid animation based on physically-based method. Hereafter, the basic of fluid simulations and animations is introduced in the section II. Section III describes about fluid control with a brief review of previous works. Then, the comparison of the different control methods is shown in the section IV and conclusion is in section $\mathrm{V}$.

\section{Basic of Fluid Simulations and Animations}

Formerly; in computer animation, the animators simulate the fluid movement by using nonphysically based method; do not solving the governing equations of fluid dynamics. For examples, random velocity field method is used to generate a 3D grid of random vectors of velocity and used them for interpolation. A vortex method is similar, but forces the velocity field to contain swirly fluid-like vortices by projecting an arbitrary velocity field into a divergence-free one [1]. Chen and da Victoria Lobo [2] proposed high field method for simulation by solving Navier-Stokes' in two-dimensional; then, push the high field up and down depended on the pressure to create the third dimensional. Witting [3] also solved Naveir-Stoke's equations in two-dimensional system and created third-dimension by using approximating scheme; forth order Runge-Kutta. Nevertheless, people have higher requirements on the visual effect of animation. The artists or animators have to spend much time on the complex scene. Various varying movements of fluid are difficult for the animators to simulate frame by frame. The alternative method for creating realistic fluid animations is Physical model. Unlike key frame or procedural based techniques, the method allows animators to effortlessly create fluid phenomena due to its governing equation which describes the fluid behaviors.

To simulate the fluid flow based on physical method, we must have a mathematics model for representing the state of the fluid at any time. The most significant properties for fluid simulation are the velocity field because we can determine how the fluid moves itself. In 1845, Navier and Stokes introduced the equations for describing the behaviors of fluid, 
called 'Navier-Stokes' equation'. Three fundamental governing the equations describe the following characteristics; the continuity indicating that the fluid mass conservation; momentum preserving Newton's second law, and energy conservation. The equations are common to make simplifying assumptions when modeling complex phenomena.

In order to solve the mentioned equations, we can achieve by using computational fluid dynamics (CFD) which is the method used for actual flow prediction. In the field of computer graphics; however, fluid simulations require far less precision than those used in physics field. Animators require the systems or tools that can be easily implemented, less computation, and give them believable result or look fairly good. So, we assume incompressible fluid which its volume in the system is constant throughout the time and homogeneous fluid whose density is constant for the simulation. The combination of incompressibility and homogeneity leads the fluid whose density and temperature are nearly constant. The assumptions are common in fluid dynamics because fluids regularly move at low speeds, whereas high speed moving phenomena are governed by other equations.

Hence, supposed incompressible and homogeneous fluid, the details of those equations are described as follow;

\subsection{Navier-Stokes' equations}

Let $\vec{u}$ is the velocity of fluid which composed of three components of $3 \mathrm{D}$ velocity $(u, v, w)$. The Naveir-Stokes' equations are usually written as

$$
\begin{gathered}
\nabla \cdot \vec{u}=0 \\
\frac{\partial \vec{u}}{\partial t}=-(\vec{u} \cdot \nabla) \vec{u}-\frac{1}{\rho} \nabla p+v \nabla^{2} \vec{u}+F
\end{gathered}
$$

Where $v$ is the kinematic viscosity, $\rho$ is the (constant) fluid density, $p$ is pressure, and $F$ is any external forces that effect on the fluid. The equation (2.3), continuity equation, state that the rate at which in any small region of fluid, the total amount of mass per volume entering is exactly equal to the amount leaving volume. Thus, mass is always conserved during flow. The equation (2.3) notifies that the fluid velocity field always has zero divergence.

Equation (2.4) states that the momentum is conserved. The equation has several components which describes the change in velocity of the fluid at a given position is related to four terms; self-convection (advection), pressure, internal resistance (diffusion), and external forces. The term on the left hand side, $\frac{\partial \vec{u}}{\partial t}$, is the time derivative of the fluid velocity. On the right hand side, it can be separated into 4 terms as the following descriptions:

Advection $((\vec{u} \cdot \nabla) \vec{u})$ : The first term represents the 'self-advection' of the velocity field which accounts for the direction (or velocity vector) which is changed by convection.

Pressure $\left(\frac{1}{\rho} \nabla p\right)$ : The second term is pressure gradient. It states that fluid particles are push in a direction from high to low pressure.

Diffusion $\left(v \nabla^{2} \vec{u}\right)$ : The third term which is called diffusion term, describes how quickly the fluid damps out variation by measuring the parameter $v$ which is represents a kinematic viscosity of the fluid. The higher its value is said that thick fluids and it flow slowly.

External Forces $(F)$ : The final term contains the external forces applied to the fluid. These forces may be either local forces or body forces. Local forces are applied to specific area whereas body forces act globally on the fluid.

However, Navier-Stoke's equations are in the form of vector field, when we are going to simulate, we have to project to other system in order to be easy to use in computation. The forms of the equations could vary according to the viewpoints of fluids such as Eulerian or Lagrangian. 


\subsection{Eulerian approach}

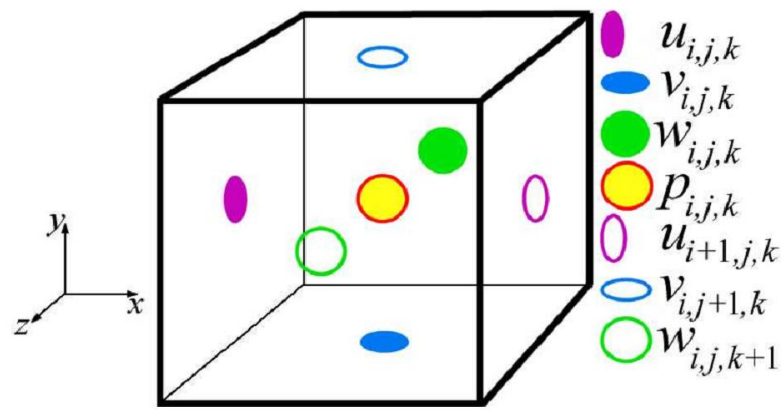

Figure 1 Maker-And-Cell method [4]

Eulerian approach is the method which uses spatial coordinates to describe the system. The workspace is discretized equally as grid. The change in system is being tracked at fixed grid points. One of the most commonly used techniques is Maker-AndCell method (MAC). This method has two major components. The first is the cells represented by uniform cubes or voxels which storing two types of variables; a scalar value and a vector value. All scalar values are contained the center of the cell while all vector values are stored on the cell faces describing $x, y, z$ components. Another component is a large collection of maker particles in the fluid that mark which cells are filled with fluid and that carry velocity to previously empty cells. A major strength of this method is that liquid is no longer constrained to be a height field, as demonstrate by the animations of pouring and splashing. [5]

Grid-based fluid simulation has been continuously been developed in computer graphics community. One of notable work was proposed by Stam [6]. An unconditionally stable model was introduced; they use a combination of a semiLagrangian scheme and implicit time integration. However, this model suffers from too much numerical dissipation which can cause visual artifact. The flow tends to dampen and vanish rapidly; thus, the small scale detail is missing.

One of the main problems of using grid based representation is the uniform grid size; the finer details are depended on grid size. Recently, many researchers proposed developed techniques based on grid-based approach to adjust the grid size, such as Octree method [7] and model reduction [8]. The high resolution data of fluid flow is refined into fluid tiles in order to capture spatially localized fluid behavior. The models are extremely fast and scalable to large domains, but cannot be used to simulate multiphase flows; moreover, fluid with free surfaces cannot be properly handled.

Although grid based method is efficient and rather fast, this approach is still difficult to implement for control. Moreover, it is also difficult to simulate small scale of details due to its scale ability.

\subsection{Lagrangian approach}

The system which is described by Lagrangian viewpoints use material coordinates to introduce the change in system is being tracked the position of each particle. In order to solve the equations by using Lagrangian approach, the continuum is treated as a particle system. Particle system was introduced by Reeves [9] in 1983. After that, this method has been widely used for modeling fuzzy objects such as fire, water, and clouds. The concept of this system is modeling an object as a large collection of simple geometric particles that define its volume and contain some attributes such as mass, density, velocity, and pressure. Over a period of time, particles are generated into the system; move and change the form within the system, and die from the system.

The particle system based interpolation method which is popular and widely used is Smoothed Particle Hydrodynamics (SPH). SPH was developed by Gingold and Monaghan [10] and Lucy [11] for the simulation of astrophysical problems and have 
been used more to study among other astrophysical topic. Hereafter, we will give a brief of the SPH in the following subsection.

\subsubsection{Smoothed particle hydrodynamics (SPH)}

Smoothed Particle Hydrodynamics (SPH) is one of the easiest particles methods. The $\mathrm{SPH}$ formalism was firstly introduced by physicists for cosmological fluid simulation in 1977. SPH was developed by Gingold and Monaghan [10] and Lucy [11] for the simulation of astrophysical problems and have been more used to study among other astrophysical topics, i.e. large scale structure in the universe, galaxy formation, supernova and solar formation. Because of its Lagrangian nature, SPH method is general enough to be adopted to solve various problems not only in computational fluid dynamics; both compressible and incompressible flow, but also in multiphase flow, heat and mass transfer, and solid mechanics.

$\mathrm{SPH}$ is an interpolation method for particle systems. In SPH, the fluid is sampled by a set of elements called particles. Each particle contains some attributes or physical properties, such as local mass, density, velocity, or pressure. With SPH, the values and derivatives of continuous physical quantities can be approximated by a set of discrete particles. To achieve this, SPH distributes quantities in a local neighborhood of each particle according to a smoothing kernel.

Beginning

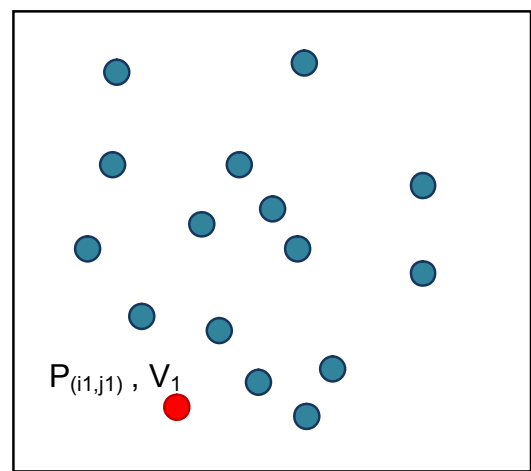

Next time step

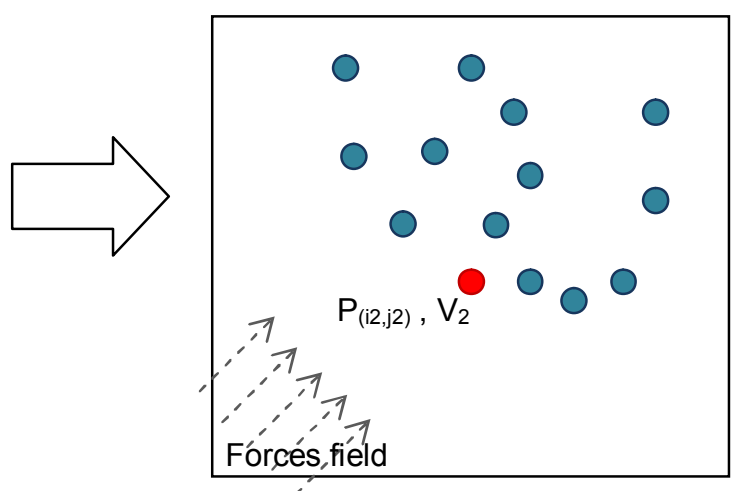

Figure 2

Particle system

- is tracked particle representing its position and velocity
Let a fluid represented by a set of particles $i \in\{1,2, \ldots ., M\}$ with position $\mathrm{x}_{i}$, masses $m_{i}$ and additional attributes $A_{i}$. A fluid property $A_{S}(\mathrm{x})$ at position $\mathrm{x}$ in space is computed by weighted sum of the fluid properties $A$ of neighboring particles within finite distance as

$$
A_{S}(\mathrm{x})=\sum_{j \in N} m_{j} \frac{A_{j}}{\rho_{j}} W\left(\mathrm{x}-\mathrm{x}_{j}, h\right)
$$

Where $A_{S}(\mathrm{x})$ is the summation of interpolated particular field variables at particle $i$. $j$ iterates over all particles which are in a set of neighbor of particle $i$ notated as $N$, and its position $\mathrm{x}_{j} . A_{j}$ is the field quantity at $\mathrm{x}_{j} . m_{j}, \rho_{j}$ are the mass and density of particle $j$ respectively.

The kernel function $W(r, h)$ is typically a radial symmetrical smoothing kernels with smoothing length, $h$. Moreover, it is normalized function with finite support, i.e. $W(r, h)=0$ for $\left|r-r_{j}\right|>h$ and $\int W(r, h) d r=1$.

This method is a particle-based method which represents sample points that enable the approximation of the values and derivative of local physical quantities inside. It is general enough to be adopted to solve various problems. Many researches use SPH method to simulate various fluid flows.

However, the capability of SPH depends on its mean values relied on kernel length. Hence, if it is defined improperly, it will spend waste time in dense areas; in the other hand, in low density areas will lack of particles for calculation. In order to solve this 
problem, there are many researches proposed the techniques; called Adaptive $\mathrm{SPH}$, for adjusting the size of particle. Size adaptation leads particle to be fit with the density of its region. The main idea is splitting and merging particle; enlarged size in dense area and reduced size in sparse area [12], [13], [14].

In another direction for solving the problem, some previous works proposed the method of adaptive SPH by adjusting the kernel length. In physical fluid dynamics field, [15], [16] provided an alternative technique to improve the performance of numerical solutions of dynamical problems. The technique provided for representing functions and derivations with adaptive parameters which can be automatically adjusted to optimal values according to the location of the particles. To couple with particles method which is based on SPH, the considerable parameter is the smoothing length or influence radius; $h$. Due to the key advance of SPH which associates with each particle is a smoothing length representing the finite spatial extent of the particle, the smoothing length (or radius) can differ in value for separate particle, as well as vary in time [17]. By varying the kernel radius, it is possible to achieve significant improvements over the fixed kernel radius approaches. Substantial improvement on the approximation of functions and derivatives are obtained by allowing the kernel parameters to dynamically accommodate, both global and local, to the all given points.

Furthermore, coupling Eulerian and Lagrangian method is an alternative technique for solving the problem due to each approach has its advantages and limitations. Many researches proposed the combined method for simulating fluid phenomena, as described in the following subsection.

\subsection{Hybrid method}

According to the different features of those principal approaches, researchers are desired to combine their advantages while avoid raising limitations. In Eulerian method, we found that it would be suitable for the simulation which has smooth surface. However, it would suffer from lengthy computation time, aliasing boundary, and poor scalability. On the other hand; in Lagrangian method, has its advantages to demonstrate the fluid phenomena with small details. The demands of computational resources of particles systems with moderated quantity of particles are generally less than its Eulerian. The key advances of SPH; however, is a smoothing kernel that should be designed carefully because of the stability, accuracy, and speed. Fixed inappropriate kernel length causes either the small details lost in the low density regions, or amount of computation time consumed. Furthermore, there are various choices of the smoothing function provided for interpolating different state of the flows and fluid attributes. In addition, this method is difficult to construct a smooth surface for rendering.

Researchers are desired to combine their advantages while avoid raising limitations by coupling the features of those two method. In the fluid system, the constraints have to be defined for separating the flow appearance; on the other word, we have to know where using grid based or a particle based for simulation. A basic idea to divide the state of the flow is grid based approach used for smooth flow and particle method implemented to catch the small details. The properties which are commonly used to identify the constraints for the flow separation are velocity, density and distance from surface.

The particle level set [18] was created to compensated for some of the inaccuracies when advecting level sets by adding Lagrangian particles to the simulation and letting them correct the first order error with the inherent high resolution detail near the front that the particles contain. Particles were used both inside and outside the surface of fluids; called interface, in order to correct the error in surface representation. In 2004, [19] proposed a method for directable animation of photorealistic liquid by using the particle level-set method. In this work, its main concept is applying different level of velocity calculation to each region which is divided due to user's specification. [20] proposed a two-way couple simulation framework that uses particle-level set method to efficiently model for simulation. The novel method allows dense areas modeled with incompressible Navier-Stokes' equations; based on grid representation, while SPH methods are used for diffuse areas. Its main limitation of the approach is that using FLIP method which introduces unwanted noise. Another limitation occurs where SPH particles do not have neighbors on all sides, so particle density estimation is unreliable near the air/liquid interface. The main advantages of using particle-level set method 
particles do not have neighbors on all sides, so particle density estimation is unreliable near the air/liquid interface. The main advantages of using particle-level set method are finer details preservation and computation time reduction. However, the limitation is that must be aware of unreliable computation of particle density nears the air or liquid interface.

Figure 3

Particle-Level Set method [20]. The level set is depicted in green while added particles are in red.

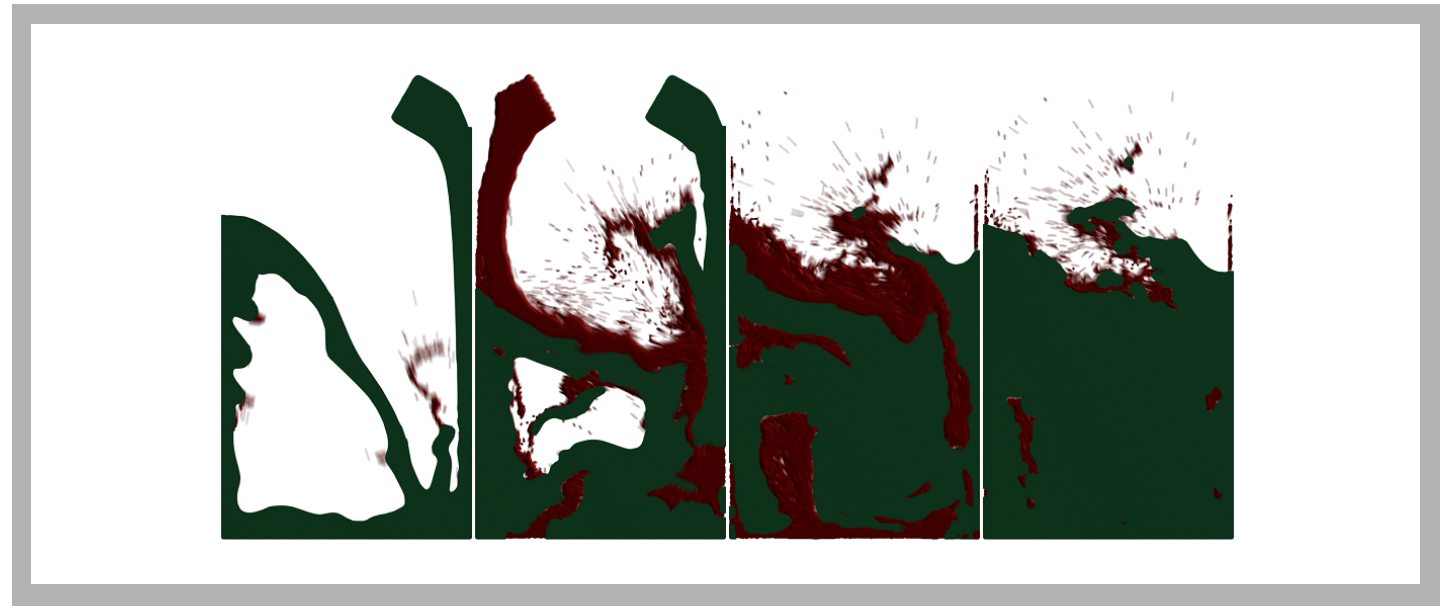

III. Controllable Fluid Animation

In the graphics community, animators not only need realistic animation, but also need a desirable fluids dynamics. They would like to control fluid behaviors as their imagination. Consequently, fluid control methods should be evaluated with the criteria; control ability, ease to use, fluid-like movement, and stability.

For control, we have to interpret the control forces in order to insert into the simulation steps. In general; the force of control is usually interpreted to one of these 3 constraints; as an external forces function, as a velocity constraint, or as a direct constraint of velocity. We have to satisfy that it has tradeoff between control and realism.

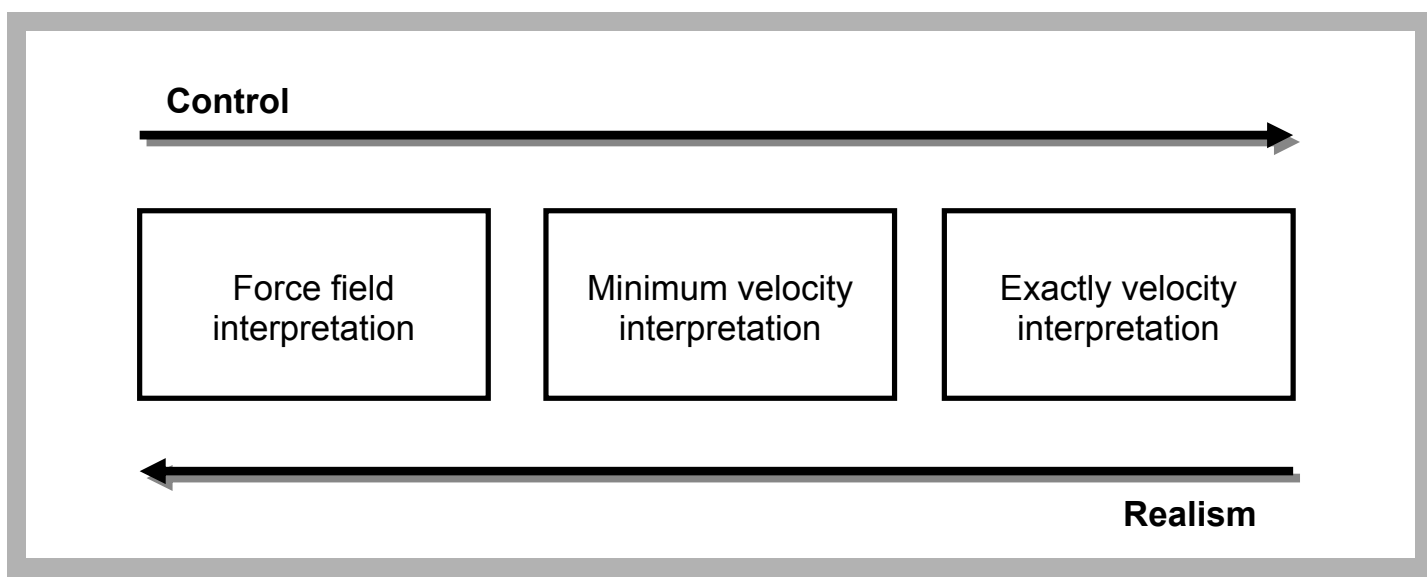

Generally, these values are changed; fluid properties, external pressure field, internal pressure field, velocity field and, boundary properties in the simulation process. Consequently, we can apply the control forces to those processes in order to control the fluid movements. Foster and Mataxas [21] firstly introduced controllers for animating phenomena. By using the concept of an embedded controller, animators can specify and control the fluid animation without knowledge of the underlying equations. The limitations of the method occur when using large time step size. Other problems are that the object have to be simple compared with gird size and the method cannot deal with transparency. Thereafter, the developed techniques for control fluid have been continuously proposed.

Recent techniques on fluids control have corresponded physic-based fluid dynamics. A way to use key-framing method of fluid motion to enhance the visual effects and user 
boundary, which are applicable to existing systems and able to combine to a variety of effects.

Firstly, we would like to introduce common defining control representations or desired fluid movements which are used with key-frame animation in computer graphics, the descriptions are in the following subsections.

\subsection{Path defining control}

To define the movement of fluids, we usually group a constrained motion into pathdefining control and object guiding control. Path defining control allows the user to specify the direction of fluid movement by a curve or a line; as show in the following figure.

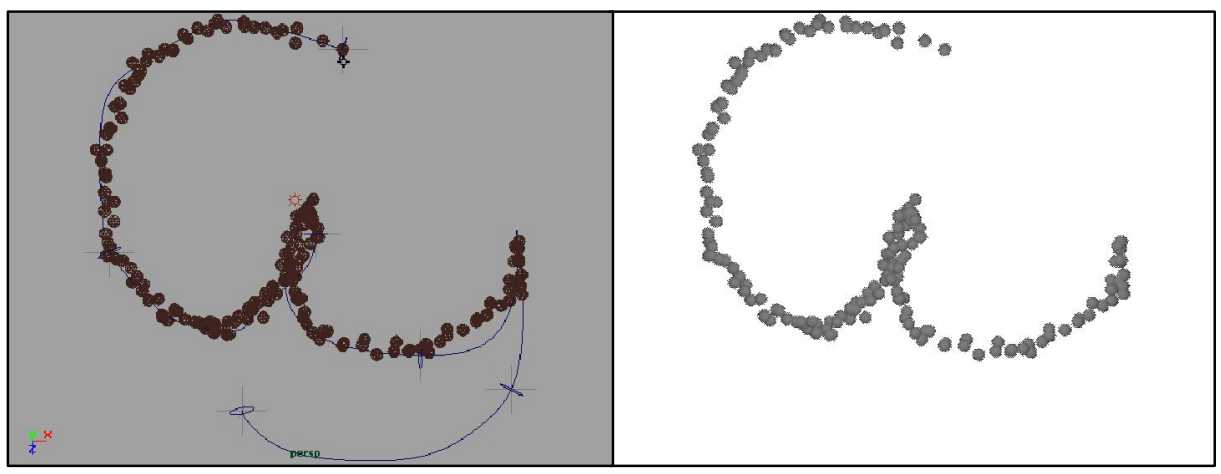

Figure 5

The example of path defining control

In general, the method which used with control path is particle control method. The method is based on particle system which defines a set of 'control particle' for leading its neighboring particles along the user-defined direction. One of the techniques for control the particle was developed by [23] based on the Advected Radial Basis Function; therefore, the local properties of the fluid are modeled by time-varying kernel. Advected particles served as center of Radial Basis Function and then used as a calculation point in SPH. The flow can be modeled and edited by using the method. However, the limitation depends on a fitting finite number of particles and fixed radius. [24] proposed the novel technique to model a controllable fluid simulation by coupling Reeb Graph and Radial Basis Function (RBF). In addition, SPH is used to approximate flow dynamics, and the concept of skeletal particles is used for controlling. However, there are visual artifacts because of averaging velocities of fluid particles with skeletal particle. Another problem which occurs in this method is that it cannot automatically choose the number and destination of skeletal particles.

\subsection{Object defining control}

As the common application in animation, the fluid usually forms into a model or the object. Therefore, another specified motion constraint as an object guiding control or target shape constrained is more used to allow the user providing the destination shape to guiding the movement of fluid flow. [25] described a method for controlling smoke simulation through user-specified key frames. This method become computationally prohibitive for large problems with fine grained control, and get caught in local minima. [26] also proposed a method for efficiently controlling animated smoke. In order to achieve, new terms are added to the standard flow equation; Navier-Stokes equation. These terms are driving force term and a smoke gathering term. Therefore, complex smoke can be controlled and easily extended to support the usual external forces. Nevertheless, this method cannot be used to control the fluid motion by userspecified as for flow-path. 
Figure 6

the control

techniques which are

based on objects

guiding,

(a), (b), (c) presented in [28], [29], [31] respectively.

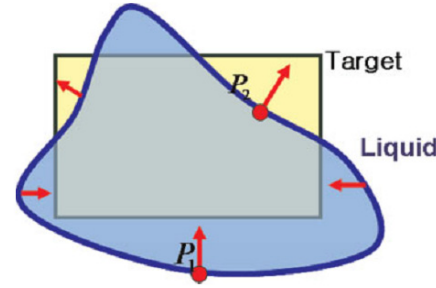

(a)

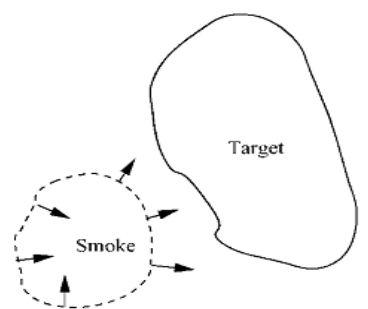

(a)

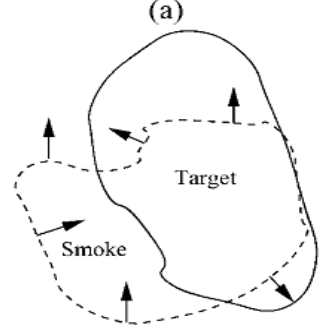

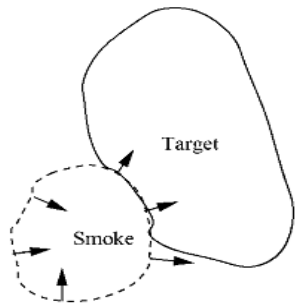

(b)

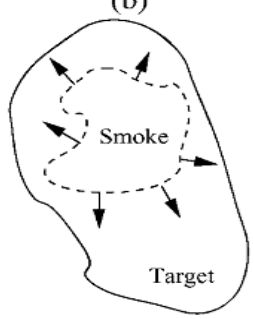

(c)

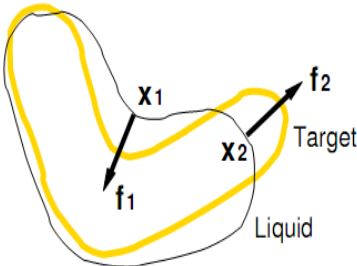

(b)

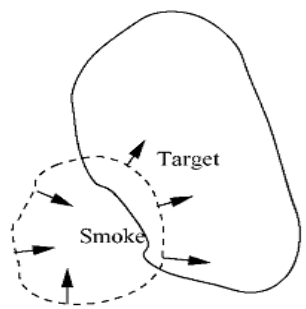

(c)

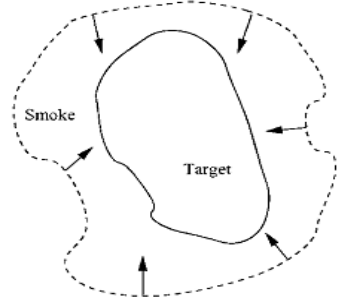

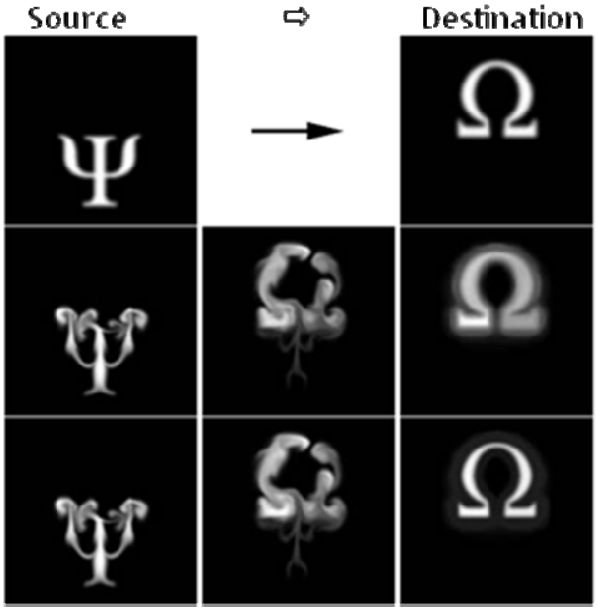

disable gathering term

enable gathering term

Control smoke

simulation [26]

Other ways for controlling fluid flow is such proposed in [27]. They used Adjoint method to control fluid. By using this method, derivations can be computed efficiently and easily handle huge control vectors. Nevertheless, discontinuities in the water simulator make control more difficult. Moreover, it is less accurate for smoke. Jeongmo Hong and Chang-Bun Kim [28] presented a new fluid control technique that uses a geometrically induced potential filed in order to preserve a geometric of the targetobject. A potential added as an extra dimension to the simulation space which forces the fluid to inform the target shape. [29] is also their proposed method for controlling liquids flow into a target shape by using a concept of shape feedback. The force is determined from the magnitude of pressure jump. Pressure jump; also used in [30], provides the force to make the fluid assuming the target shape. Instead of adding the force to an external term, it is added at the projection step. The drawback of this method is the forces may cause visual artifact. Another method which based on feedback control forces was proposed by Lin Shi and Yizhou Yu [31]. They introduce an efficient and effective solution applying the external forces field which composed of 
velocity and shape feedback. It makes use of continuous sequence of frames. However, the method does not support some interesting phenomena such as splashing. [32] proposed the method which uses imposing velocity constraints. The smoke shapes are created resembling to user-specified objects and, both smoke region and the target object are represented by implicit function. The velocities constraints are derived from shape matching process. The advantages of this method trade of between controllable and smoke appearance.

\section{Comparisons of Different Control Types}

According to the users' requirements, as physically based fluid animation, there are not only realistic appearances and plausible visual effects be the significant criteria, but also the control ability such as shown in section III. Moreover, the computation time is also considered. There is no clear which introduced control method is the best choice for every simulation since each method has its advantages and limitations.

\subsection{Different types of control method}

The path defining control uses the user-specified line or curve to control the direction of fluid movement. Therefore, it is easy to apply to the free surface flow. Moreover, this method can be applied to interactive simulation. The path defining control is effortless to apply with particle based simulation more than grid based representation because fluid boundary is not clear. However, the limitation of particle control method is how to find the position and number of control points.

The major advantage of the object guiding control method is preserving a geometric of the target-object. This method can make sure that the target object full filled with fluid. Moreover, by using the features of target shape as an interface or a surface boundary of fluid, many proposed techniques such as [29], [30], [31] use the semi-Lagrangian particle level-set method for simulation. This method leads to fast computation because of grid based representation. Nevertheless, the efficiency of the result depends on userdefined constraints such as target driven forces. These forces may cause some artifact; for example, the resulting smoke transition does not come across as a natural evolution of smoke. Skill and experiments are important role due to the right target states and appropriated control parameters are chosen for a good animation.

\subsection{Different types of fluids}

There are a lot of fluid types in the nature which have different characteristics and its visual effects. For example, smoke and cloud have small viscosity and do not hold a clear surface; as a result, there are some constraints for confinement such in [33] which included vorticity confinement force. Another purposed method such in [26]; in order to gather the smoke in the guiding shape, gathering term is a new term added in the Navier-Stokes' equation as a driving force for control. Moreover, the shape or interface of smoke can be clearer by adjusting the parameter.

Another kind of fluid is such mud, gel, and so on, which display variable viscosity. The interface or surface of those fluids is clear to define the shape; therefore, the application is commonly intent to capability of control such proposed in [27]. By the way, pathdefining control easily applies to the viscous flow because it flows clearly through along the path direction.

\section{Current research areas and directions}

Due to the differences of behaviors and visual effects of the types of fluid, various control methods, and several of requirements, there are modifications in many ways to satisfy the results. In this section, we will briefly review some research areas and direction in the recent years.

\subsection{Constraints consideration}

There are various constraints which are used for control the movement of fluid in order to support the requirement of user. The control constraints can be implied from the method which is used for control such as key-frames. [22] introduced the advantages of using key-frames for simulation. Three general types of key-framing; position to 


\subsection{Constraints consideration}

There are various constraints which are used for control the movement of fluid in order to support the requirement of user. The control constraints can be implied from the method which is used for control such as key-frames. [22] introduced the advantages of using key-frames for simulation. Three general types of key-framing; position to position, density to density, and boundary to boundary, are applicable to existing systems and able to combine to a variety of effects. The researches which make use of key-frame control coupled with guiding object such as shown in [25], [26], [28]-[32]. Generally, the shape of guiding object is very useful for defining the control forces. In addition, some researches utilized the advantage from path definition i.e. [25] use the skeletal which obtained by extracting the skeleton of guiding object to control the fluid movement. This approach derives the representation of object to a path which is easier for calculation. Thus, the purposed method for representing the object in the simulation is one of interesting challenges for researcher.

\subsection{Physical model modification}

As show in the Navier-Stokes' equation, there are 3 terms that affect to the differentiation of velocity; the term of pressure, diffusion and external forces. The control forces can be interpreted to apply to those terms in the computation steps. Mostly, the interpretation simultaneously use with the concept of shape feedback, as show in [29]-[32]. The control forces are determined from the shape, and then projected to compose the velocity. Moreover, the additional term for control or preserve the quality of visualization can be also applied to the Navier-Stoke's equation i.e. gathering term for smoke simulation [26]. However, the efficiency of these approaches depends on appropriated control parameter which obtained from experiments.

\subsection{Interaction (interact with user)}

Recently, the application for control fluid which is real-time interactive with user is interested. Some researches proposed the method to reduce the model for calculation by separating the model into small parts which are relative by the constraints. [35] creates separated models for the velocity field and for each moving boundary which is enable large scale, real-time, detailed flows with continuous user interaction. Similarly, [36] construct a set of reduced models or tiles, which is able to compose and capture spatially localized fluid behavior.

\section{Conclusion}

Currently, control fluid animation is widely used in the graphics community, mainly in commercial such as movie, games and advertisement. In this reviews, we have introduced the basic approaches for simulating controllable fluid, the commonly constrained motion. This paper is proposed for providing the readers a preliminary briefing of the topics' references so that the beginner might easily perceive the concepts and point out the strengths and deficiencies of each approach. 


\section{REFERENCES}

[1] M. Kass and G. Miller, "Rapid, stable fluid dynamics for computer graphics," Computer Graphics, vol. 24, pp. 49-57, 1990.

[2] J. X. Chen and N. D. V. Lobo, "Toward interactive-rate simulation of fluids with moving obstacles using Navier-Stokes's equations," Graphical Models and Image Processing, vol. 57, no. 2, pp. 107-116, 1995.

[3] P. Witting, "Computatinal fluid dynamics in a traditional animation envieronment," in Proceedings of SIGGRAPH 99, Computer Graphics Proceedings, Annual Conference Series, pp. 129-136, 1999.

[4] M.T. Carlson. Rigid, melting, and flowing fluid. Doctoral dissertation, College of Computing Geogia Institue of Technology, 2004.

[5] J. E. Welch, F. H. Harlow, J .P. Shannon, and B. J. Daly, "THE MAC METHOD: a computing technique for solving viscous, incompressible, transient fluid-flow problems involving free surfaces," Report LA-3425, Los Alamos Scientific Laboratory, 1965.

[6] J. Stam, "Stable fluids," in Proceedings of SIGGRAPH 99, Computer Graphics Proceedings, Annual Conference Series, pp. 121-128, 1999.

[7] F. Losasso, F. Gibou, and R. Fedkiw, "Simulating water and smoke with an octree data structure," ACM Transactions on Graphics, vol. 23, no. 3, pp. 457-462, 2004.

[8] L. Shi and Y. Yu, "Visual smoke simulation with adaptive octree refinement," Technical Report. UIUCDCS-R-2002-2311, University of ILlinois at Urbana-Chaampaign, 2002.

[9] W.T. Reeves, "Particle system - a technique for modeling a class of fuzzy objects," ACM Transaction on Graphics, vol.2, no. 2, pp. 91108, 1983.

[10] R. A. Gingold, and J. J. Monaghan, "Smoothed particle hydrodynamics," Monthly Notices of the Royal Astoronomical Society, vol.181, pp. 375-389, 1977.

[11] L. B. Lucy, "A numerical approach to the testing of the fission hypothesis," Astronomical Journal, vol.82, pp. 1013-1024, 1977.

[12] W. Hong, D. H. House, and J. Keyser, "Adaptive particles for incompressible fluid simulation," The Visual Computer: International Journal of Computer Graphics, vol. 24, no. 7, pp. 535-543, 2008.

[13] B. Adams, M. Pauly, R. Keiser, and L.J., Guibas, "Adaptively sampled particle fluids," ACM Transaction on Graphics, vol.26, no.3, pp.48-1-48-7, Jul. 2007.

[14] H. Yan, Z. Wang, J. He, X. Chen, C. Wang, and Q. Peng, "Real-time fluid simulation with adaptive SPH," Computer Animation and Virtual Worlds, vol.20, no. 2-3, pp.417-426, 2009.

[15] Di. G.S. Leonardo, L. Hender, A. Donoso, E. Sira, and J. Klapp, "A shock-capturing SPH scheme based on adaptive kernel estimation," Journal of computational Physics, vol. 212, no. 1, pp. 124-149. 2005.

[16] Di. G. S. Leonardo and L. Hender, "Adaptive kernel estimation and SPH tensile instability," Computers and Mathematics with Applications, vol. 55, no. 1, pp.23-50, 2007.

[17] R. E. Attwood, S. P. Goodwin, and A. P. Whitworth, "Adaptive smoothing lengths in SPH," Astronomy \& Astrophysics manuscript no. Scoustic, pp.1-5, 2008.

[18] D. Enright, F. Loasasso, and R. Fedkiw, "A fast and accurate semi-lagrangian particle level set method," Computer and Structures, vol. 83, no. 6-7, pp.479-490, 2004.

[19] N. Ramussen, D. Enright, D. Nguyen, S. Marino, N. Summer, W. Geiger, S. Hoon, and R. Fedkiw, "Directable photorealistic liquids," in Eurographics/ACM SIGGRAPH Symposium on Computer Animation, pp. 193-202, 2004.

[20] F. Losasso, J. O. Talton, N. Kwatra, and R. Fedkiw, "Two-way coupled SPH and particle level set fluid simulation," IEEE Transactions on Visualization and Computer Graphics, vol.14, no. 4, pp. 797-804, 2008.

[21] N. Foster and D. Metaxas, "Controlling fluid animation," in Proceedings CGI'97, pp.178-188, 1997.

[22] M. D. Brent and K. John, "Keyframing particles of physically based systems," Theory and Practice of Computer Graphics, pp. 11-18, 2005.

[23] F. Pighin, J. M. Cohen, and M. Shah, "Modeling and editing flows using advected radial basis functions," in Eurographics/ACM SIGGRAPH Symposium on Computer Animation, pp. 223-232, 2004.

[24] S. Arisara and K. Pizzanu, "Keyframe control of fluid simulation using skeletal particles," in The Internaltional Conference of Computational Methods, International Conferencs Center Hiroshima, Japan, 2007.

[25] A. Treuille, A. Mcnamara, Z. Popovic, and J. Stam, "Keyframe control of smoke simulations," ACM Transactions on Graphics, vol. 22, no. 3, pp.716-723, 2003.

[26] R. Fattal and D. Lischinski, "Target-driven smoke animation," International Conference on Computer Graphics and Interactive Techniques, ACM SIGGRAPH 2004, pp.441-448, 2004.

[27] A. McNamara, A.Treuille, Z. Popovic, and J. Stam, "Fluid control using the adjoint method," ACM Transactions on Graphics, vol.23, no. 3, pp. 449-456, 2004.

[28] H. S. Seung, L. Jung, J. K. Sun, and H. K. Chan, "Controlling fluid animation with geometric potential," Computer Animation and Virtual Worlds, vol.15, no. 3-4, pp.147-157, 2004.

[29] S. H. Shin and C. H. Kim, "Target-driven liquid animation with interfacial discontinuities," Computer Animation and Virtual Worlds, vol.18, no. 4-5, pp.447-453, 2007.

[30] S. H. Shin, J. Lee, S. J. Kim, and C. H. Kim, "Controlling liquids using pressure jump," International Conference on Computer Graphics and Interactive Techniques ACM SIGGRAPH 2006 Sketches, Article no.: 62, 2006.

[31] L. Shi and Y.Yu, "Taming liquid for rapidly changing targets," Eurographics/ACM SIGGRAPH Symposium on Computer Animation, pp.229-236, 2005.

[32] L. Shi and Y. Yu, "Controllable smoke animation with guiding objects," ACM Transaction on Graphics, vol.24, no. 1, pp.140-164. 2005.

[33] R. Fedkiw, J. Stam, and H. W. Jensen, "Visual simulation of smoke," Proceedings of the 28th annual conference on Computer graphics and interactive techniques, ACM, pp. 15-22, 2001.

[34] A. Treuille, A. Lewis, and Z. Popović , "Model reduction for real-time fluids," International Conference on Computer Graphics and Interactive Techniques, ACM SIGGRAPH 2006, pp. 826-834, 2006.

[35] M. Wicke, M. Stanton, and A.Treuille, "Modular bases for fluid dynamics," International Conference on Computer Graphics and Interactive Techniques, ACM SIGGRAPH 2009, Article no. 39 , 2009. 